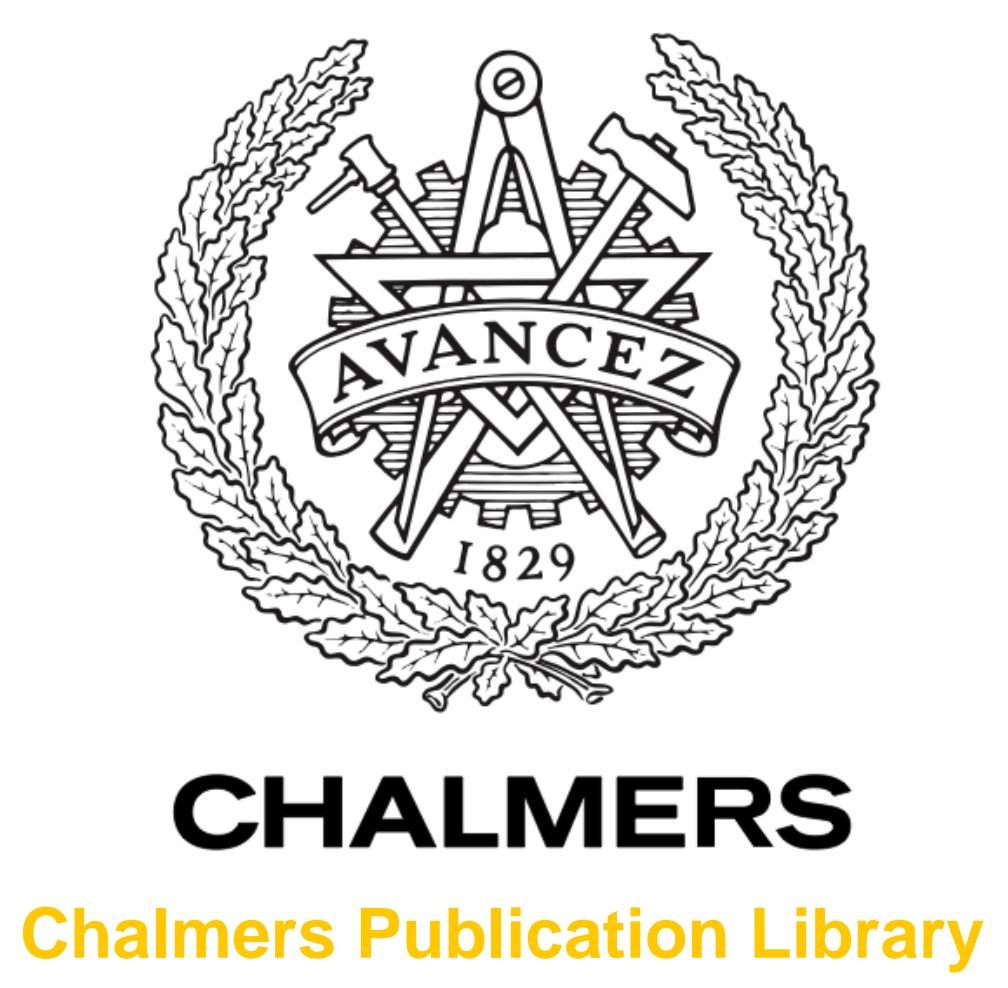

\title{
MIMO Truncated Shannon Bound for System Level Capacity Evaluation of Wireless Networks
}

This document has been downloaded from Chalmers Publication Library (CPL). It is the author's version of a work that was accepted for publication in:

IEEE Wireless Communications and Networking Conference, Paris, France, Apr. 2012

Citation for the published paper:

Burr, A. ; Papadogiannis, A. ; Jiang, T. (2012) "MIMO Truncated Shannon Bound for System Level Capacity Evaluation of Wireless Networks". IEEE Wireless Communications and Networking Conference, Paris, France, Apr. 2012

http://dx.doi.org/10.1109/WCNCW.2012.6215504

Downloaded from: http://publications.lib.chalmers.se/publication/154642

Notice: Changes introduced as a result of publishing processes such as copy-editing and formatting may not be reflected in this document. For a definitive version of this work, please refer to the published source. Please note that access to the published version might require a subscription. 


\title{
MIMO Truncated Shannon bound for system level capacity evaluation of wireless networks
}

\author{
Alister Burr ${ }^{*}$, Agisilaos Papadogiannis ${ }^{+}$and Tao Jiang ${ }^{*}$ \\ *University of York, UK and ${ }^{+}$Chalmers University, Sweden \\ agb1@ohm.york.ac.uk
}

\begin{abstract}
We outline a general method for modelling the capacity of a MIMO link within a wireless, assuming that capacity of a link is a random function of SNR and signal to interference ratio (SIR), since the maximum link throughput depends on the random channel of both the user's signal and the interference. We show how a look-up table for the CDF of this random function can be obtained by link-level simulation in the presence of interference having the same characteristics as the interference found in the target network. We also exploit the Truncated Shannon Bound (TSB) to estimate the resulting capacity obtained in practice in a system using adaptive modulation and coding on the link level.
\end{abstract}

Index Terms-MIMO; system-level simulation, truncated Shannon bound

\section{INTRODUCTION}

The evaluation of the user capacity of a cellular wireless network by computer simulation has a long history - see [1] [2] for some early examples. With the advent of LTE wireless networks it has more recently again become important - see for example [3,4]. First it is necessary to take into account intercellular interference, since this is usually the limiting factor. The performance of individual links can then be evaluated in the presence of this interference, as well as of factors such as thermal noise, Rayleigh fading and shadowing. However for a system which may contain many cells and many users per cell it is clearly not feasible to carry out a full-scale Monte Carlo simulation to evaluate the BER of every link in the system, so the approach developed in [2], for example, made use of a two-step process: a system-level simulation to evaluate the signal to interference-plus-noise ratio (SINR) of each link, taking into account path loss, shadowing and slow Rayleigh fading on both signal and interference links. Thereupon a link-level function could be applied to evaluate the capacity of the links. This could be based on the Shannon bound, or on closed-form expressions for BER versus SNR, or a lookup table obtained by simulation. In all these cases, once slow Rayleigh fading had been taken into account, this function could be treated as deterministic. (Fast Rayleigh fading could be included in the average BER function).

The introduction of MIMO, however, has made this process significantly more complicated. First, it is no longer sufficient simply to consider the aggregate interference experienced by a specific link and quantify it as a simple SINR, since the direction of the interference becomes important. And secondly, the link capacity can no longer be treated as a deterministic function of any SINR, even in the absence of interference, since capacity is a function of the channel matrix $\mathbf{H}$, which must be treated as random.

Moreover since the time of the initial system capacity evaluations adaptive modulation and coding (AMC) has become commonplace. (It is interesting to note that one of the results of these capacity evaluations was to point out the advantages of AMC as compared to the fixed modulation schemes then in use). This means that link capacity can now be treated as a function of SINR. It has been widely noted that this function can be approximated by a curve derived from the Shannon bound, either by shifting it to the right by a few $\mathrm{dB}$, or by compressing its vertical scale, or both. This has led to the concept of the truncated Shannon bound (TSB), which has been applied in 3GPP [5] to provide a simple estimate of link capacity.

In this contribution we describe a stochastically based methodology, which we have already employed within the BuNGee project, for use in a system-level capacity simulation for a MIMO network with wireless backhaul. Here we illustrate the approach using a relatively straightforward MIMO transmission scheme - spatial multiplexing with MMSE detection - the extension of the work to other schemes, such as successive interference cancellation (SIC) will be considered in future work. It exploits the TSB, and the contribution also extends the TSB concept and discusses how to match the parameters of the TSB to the performance of an actual AMC system. This discussion occurs in the next section; then in section 3 the MIMO modelling methodology is outlined, and in section 4 it is further illustrated with reference to an example of a simple MIMO transmission scheme. Finally we draw some conclusions and outline further developments that may be required for the methodology. 


\section{TRUNCATED SHANNON BOUND}

The Shannon bound gives an upper limit on the throughput of a communication link of bandwidth $W$ and signal to noise ratio $S / N$ :

$$
C=W \log _{2}(1+S / N)
$$

The state of the art in modulation and coding schemes is of course a few $\mathrm{dB}$ from this bound. Much of this gap is irreducible, since the Shannon bound can be reached only in the limit of infinite block length and infinite decoding complexity. It is approached most closely by iterativelydecodable codes, notably turbo and LDPC codes. The area property of the EXIT chart determining the convergence of such decoders implies that as capacity approaches the Shannon bound the number of iterations tends to infinity. Moreover the dominant form of coded modulation, bit interleaved coded modulation (BICM) [6], is known not to meet the Shannon bound in general, and this tends to mean in practice that the gap to Shannon is greater at higher spectrum efficiencies. Hence the throughput of practical modulation and coding schemes, a subset of which is used in wireless standards like 3GPP LTE/LTE-A and WiMAX, can in general be approximated by a function of similar shape to the Shannon bound, but shifted to the right and/or compressed on the vertical scale by a scaling factor less than 1 . Figure 1 shows the comparison between the throughput function given by the specific set of coding/modulation schemes used in BuNGee [7] and a version of the Shannon bound shifted by $1.2 \mathrm{~dB}$ and scaled by 0.82 . The upper vertices of the steps of the throughput function denote the throughputs and required SNRs for the set of schemes.

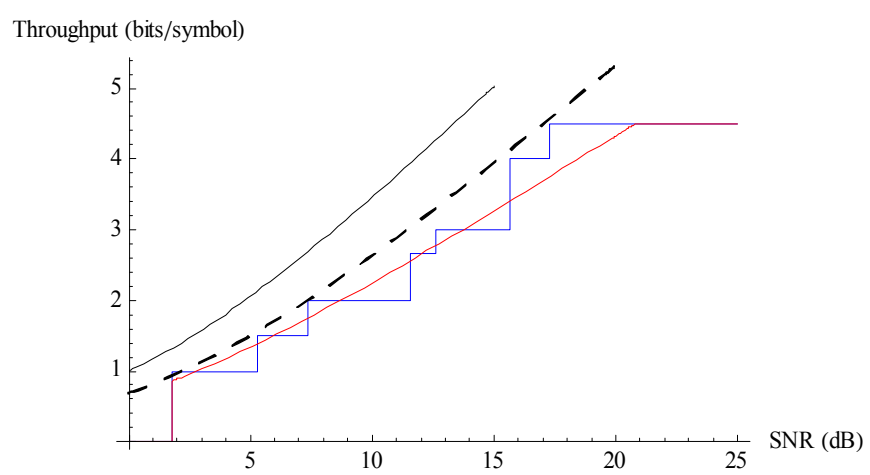

Figure 1 Throughput function of a set of coding/modulation schemes (stepped blue) compared with Shannon bound (solid black), shifted/scaled Shannon bound (dashed black), and truncated Shannon bound (red)

However this approximation does not take into account the granularity of the discrete set of schemes employed, which means that the true throughput function is stepped, as shown. This will further reduce the throughput achieved on average. Moreover in practice there is an upper limit to the throughput, $C_{\max }$, set by the throughput of the highest rate coding/modulation, and a lower limit $\gamma_{0}$ on SNR below which the throughput is zero, set by the required SNR of the lowest rate scheme. Hence the curve should be truncated at these points, as shown in Figure 1, as well as further scaled to account for the average throughput, leading to the truncated Shannon bound, which can be defined as:

$$
C_{T S B}(\gamma)=\left\{\begin{array}{cc}
0 & \gamma<\gamma_{0} \\
W \alpha \log _{2}\left(1+\gamma / \gamma_{s h}\right) & \gamma_{0} \leq \gamma<\gamma_{\max } \\
C_{\max } & \gamma_{\max }<\gamma
\end{array}\right.
$$

where $\gamma_{s h}$ denotes the shift, which we introduce here in addition to the scaling described in [5]. A question then arises of what shift and scaling parameters should be used in the TSB to optimally approximate the actual throughput function for an arbitrary set of coding/modulation schemes. In our context we wish to ensure that the average throughput given by the TSB is as close as possible to the average throughput using the true throughput function, given that the link SNR is random, so that the average should be taken over its distribution. Mathematically we can therefore write:

$$
\int_{s_{0}}^{\infty} C_{T S B}(s) p(s, \bar{s}) d s \equiv \int_{s_{0}}^{\infty} C_{t h r}(s) p(s, \bar{s}) d s=\bar{C}(\bar{s})
$$

where $C_{T S B}$ denotes the TSB function, $s=10 \log _{10}(\gamma)$ and $\bar{s}$ denote respectively the instantaneous $\mathrm{SNR}$ and the average $\mathrm{SNR}$, both in $\mathrm{dB}, p(s, \bar{s})$ denotes the probability density function of the instantaneous SNR (in $\mathrm{dB}$ ) on a given link, $C_{t h r}$ denotes the true throughput function, and $\bar{C}(\bar{s})$ denotes the average throughput as a function of average SNR. The parameters of the TSB are to be chosen to fulfil this identity, as far as possible with any likely SNR distribution.

We can approximate the PDF $p(s, \bar{s})$ with arbitrary accuracy as the sum of a set of rectangular functions: that is, the distribution can be treated as a mixture distribution of uniform distributions, provided the range $\delta$ of the support of these PDFs is less than that of the actual distribution. We write:

$$
p_{a p}(s, \bar{s})=\sum_{i=1}^{n_{p}} a_{i} u\left(s, \bar{s}_{i}, \delta_{i}\right)
$$

where:

$$
u(s, \bar{s}, \delta)=\left\{\begin{array}{cc}
0 & s \leq \bar{s}-\delta \\
\frac{1}{\delta} & \bar{s}-\delta<s<\bar{s}+\delta \\
0 & s \geq \bar{s}+\delta
\end{array}\right.
$$

Then:

$$
\begin{aligned}
\bar{C}(\bar{s}) & =\int_{0}^{\infty} \sum_{i=1}^{\infty} a_{i} u\left(s, \bar{s}_{i}, \delta\right) C_{T S B}(s) d s \equiv \int_{0}^{\infty} \sum_{i=1}^{n_{p}} a_{i} u\left(s, \overline{s_{i}}, \delta\right) C_{t h r}(s) d s \\
& =\sum_{i=1}^{n_{p}} a_{i} \int_{\bar{s}_{i}-\delta / 2}^{\bar{s}_{i}+\delta / 2} C_{T S B}(s) d s \equiv \sum_{i=1}^{n_{p}} a_{\bar{s}_{\bar{i}}} \int_{\bar{s}_{i}+\delta / 2}^{\sigma_{i}+\delta / 2} C_{t h r}(s) d s
\end{aligned}
$$

and hence capacities will match provided:

$$
\int_{\bar{s}_{i}-\delta / 2}^{\bar{s}_{i}+\delta / 2} C_{T S B}(s) d s \equiv \int_{\bar{s}_{i}-\delta / 2}^{\bar{s}_{i}+\delta / 2} C_{t h r}(s) d s, \forall \bar{s}_{i}
$$




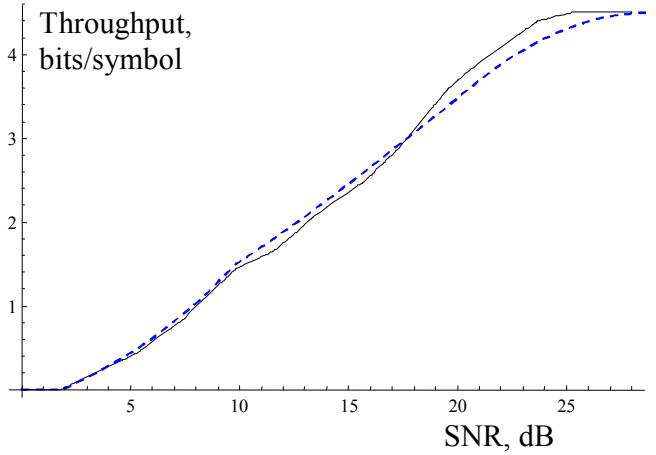

Figure 2 Average throughput against average SNR for TSB, $\alpha$ $=0.65$, shift $0 \mathrm{~dB}$ (dashed line) compared with actual throughput function (solid)

The comparison is shown in Figure 2 for $\alpha=0.65$ and zero shift.

\section{MIMO LINK CAPACITY SIMULATION}

We now consider the capacity of a MIMO link, and develop a stochastic model for it which can readily be used in a system-level simulation. Since the capacity is in fact random, depending on the MIMO channel matrix, for Monte Carlo simulation at the system level, we need to generate random instances of the capacity which follow the statistics of the capacity of the typical link, making use of parameters for the link which can be obtained from the system-level simulation. The most important such parameter is the signal to noise-plus-interference ratio encountered at the receiver of the link. We will see, however, that in many cases this single parameter does not fully define the link capacity distribution: it may depend on other factors such as the balance of noise and interference, the number of interferers and their directions.

In SISO systems we can directly apply the TSB, as described above, to estimate the throughput as a function of SINR, which can then be expressed in closed form. In MIMO systems the process is not usually as straightforward. Here we consider a class of schemes in which linear or nonlinear processing is used at the receive and/or transmitter to provide one or more streams over which conventional AMC can be used. This is a broad class, encompassing space time block codes (STBC), spatial multiplexing using both linear receivers (MMSE or ZF) and non-linear (successive interference cancellation - SIC), and precoding. The TSB can then be applied based on the SNR experienced on each stream, and an aggregate link throughput can thus be obtained.

It is however very difficult in general to obtain the distribution of the stream SNR in closed form for most schemes, especially for realistic channel models, and hence in general link-level Monte Carlo simulation has to be used to estimate the distribution of stream SNR and hence of throughput. This should then be stored in a look-up table from which random instances of link throughput can be drawn.
A convenient format for this look-up table is the cumulative distribution function (CDF) of throughput, defined as:

$$
F_{C}(\tilde{C}, \bar{s})=\operatorname{Pr}[C \leq \tilde{C} \mid \bar{s}]=\int_{0}^{\tilde{c}} p(C, \bar{s}) d C
$$

where $\bar{s}$ denotes the average SNR in $\mathrm{dB}$. This is a monotonically increasing function within the range $[0,1]$. We may then generate a random variable $f \in[0,1]$ with uniform distribution, and use it to look up $\tilde{C}$ such that $F_{C}(\tilde{C}, \bar{s})=f$. The PDF of $\tilde{C}$ is then given by:

$$
\begin{aligned}
p_{\tilde{C}}(\tilde{C}) & =\underset{\delta C \rightarrow 0}{\operatorname{Lt}}\left[\frac{\operatorname{Pr}[\tilde{C} \in \delta C]}{\delta C}\right]=\underset{\delta C \rightarrow 0}{\operatorname{Lt}}\left[\frac{\operatorname{Pr}\left[F_{C} \in \delta F_{C}\right]}{\delta C}\right] \\
& =\operatorname{Lt}_{\delta C \rightarrow 0}\left[\frac{\delta F_{C} p\left(F_{C}\right)}{\delta C}\right]=\frac{d F_{C}}{d \tilde{C}}=p_{C}(\tilde{C})
\end{aligned}
$$

Hence the random variable $\tilde{C}$ has the required distribution. Since this CDF is in general also a function of the average SINR $\bar{s}$, a two-dimensional look up table is required. If other factors such as INR, etc, are also significant, then a larger number of dimensions may be required. However if the effect of such factors is not large, or if their distributions are separable from that of SINR, a good approximation is obtained by averaging over them.

\section{EXAMPLE: SPATIAL MULTIPLEXING WITH MMSE DETECTION}

In this section we illustrate the method with reference to MMSE detection of spatial multiplexing in the presence of interference. We assume all terminals are equipped with uniform linear antenna arrays, and we use a finite scattering channel model [8] for both signal and interfering links. This explicitly takes into account directions of multipath components corresponding to both signal and interferers.

The MMSE detection technique is described in [9]. We assume that the number of receive antennas, $n_{R}$ is equal to the number of transmit antennas $n_{T}$ on the user terminal, while there may be one or more interfering users, also with the same number of transmit antennas. Hence the MMSE detector is able to recover the wanted signal, but does not have sufficient degrees of freedom to null the interfering signals. However, since in this case the interference may be correlated at the receiver, the MMSE detector may exploit this correlation to minimise the interference at the detector output. We suppose that an MMSE filter $\mathbf{W}^{H}$ is applied to the received signal vector $\mathbf{y}$, where it may be shown that to minimise mean square error, $\mathbf{W}$ should be given by [7]:

$$
\mathbf{W}=\left(\mathbf{H H}^{H}+\frac{P_{i n t}}{n_{T} n_{\text {int }}} \mathbf{H}_{\text {int }} \mathbf{H}_{\text {int }}^{H}+\sigma^{2} \mathbf{I}\right)^{-1} \mathbf{H}
$$

Here $\mathbf{H}$ denotes the $\left(n_{R} \times n_{T}\right)$ channel matrix for the signal, and $\mathbf{H}_{\text {int }}$ denotes the $\left(n_{R} \times n_{T} n_{\text {int }}\right)$ composite matrix for the 
interference from $n_{\text {int }}$ interferers each with $n_{T}$ transmit antennas. Since in spatial multiplexing the signals on these interfering transmit antennas are statistically independent, this is equivalent to the interference from $n_{T} n_{\text {int }}$ separate antennas, which can be modelled using a single matrix. Assuming that the total transmitted signal power is unity, $P_{\text {int }}$ denotes the total interference power from all interfering antennas, and $\sigma^{2}$ denotes the noise power per receive antenna. Hence the SNR is $1 / \sigma^{2}$, the SIR is $1 / P_{\text {int }}$, and the SINR is $1 /\left(\sigma^{2}+P_{i n t}\right)$.

The finite scattering channel model [8] accounts explicitly for the multipath components for each link, assigning directions of departure at the transmitter, and of arrival at the receiver, as well as fade coefficients for each multipath. The channel matrix may be written [8]:

$$
\mathbf{H}=\boldsymbol{\Psi}_{R} \boldsymbol{\Xi} \boldsymbol{\Psi}_{T}^{H}
$$

where $\boldsymbol{\Psi}_{R}, \boldsymbol{\Psi}_{T}$ are the $\left(n_{R} \times n_{S}\right)$ and $\left(n_{T} \times n_{S}\right)$ steering vector matrices at the receiver and transmitter respectively, that is, their $i^{\text {th }}$ columns are formed by the steering vector at the respective antenna array for the $i^{\text {th }}$ multipath component:

$$
\boldsymbol{\psi}_{\{R, T\}}=\exp \left(2 \pi j l_{\{R, T\}} \mathbf{k} \sin \left(\phi_{\{R, T\} i}\right)\right)
$$

where $l_{\{R, T\}}$ denotes the element spacing, in wavelengths, at the receive/transmit antenna array, $\mathbf{k}=\left\{0,1 \ldots n_{\{R, T\}}-1\right\}$ is a vector of indices of the elements at the receive/transmit array, and $\phi_{\{R, T\} i}$ is the direction of arrival/departure of the $i^{\text {th }}$ multipath at the receive/transmit array. The column index, $i=1 \ldots n_{S}$, where $n_{S}$ is the number of significant multipaths. $\boldsymbol{\Xi}$ is an $\left(n_{S} \times n_{S}\right)$ diagonal matrix whose $i^{\text {th }}$ diagonal element $\xi_{i}$ is the fade coefficient of the $i^{\text {th }}$ multipath. Independent Rayleigh fading of the multipaths is assumed, and hence the $\xi$ 's are independent complex Gaussian variables, with mean square magnitude $1 / n_{S}$. This also implies that the mean square amplitude of the elements of $\mathbf{H}$ is unity.

The MMSE detector reconstructs the transmitted signal at its output, with distortion due to noise, interference, and the reconstruction error of the MMSE detector. The total squared error can be written [7]:

$$
\begin{aligned}
\sigma_{\text {err }}^{2} & =\sigma^{2} \operatorname{trace}\left(\mathbf{W}^{H} \mathbf{W}\right)+\frac{P_{i n t}}{n_{T} n_{\text {int }}} \operatorname{trace}\left(\mathbf{W}^{H} \mathbf{H}_{\text {int }} \mathbf{H}_{\text {int }}^{H} \mathbf{W}\right) \\
& +\frac{1}{n_{T}} \operatorname{trace}\left((\mathbf{Q}+\mathbf{I})^{-1}\right)
\end{aligned}
$$

where:

$$
\mathbf{Q}=\frac{1}{n_{T}} \mathbf{H}^{H}\left(\frac{P_{i n t}}{n_{T} n_{i n t}} \mathbf{H}_{i n t} \mathbf{H}_{i n t}^{H}+\sigma^{2} \mathbf{I}\right)^{-1} \mathbf{H}
$$

Then the signal to noise ratio $\gamma$ after the detector is $1 / \sigma_{\text {err }}^{2}$. This can be used in the TSB to estimate the capacity in bits/symbol. Since in a spatial multiplexing system $n_{T}$ symbols may be transmitted per channel use, this should then be multiplied by $n_{T}$ to give the MIMO link throughput.

Figure 3 shows the effect of different levels of interference, given in terms of the interference to noise ratio (INR). For given SINR there is a variation in throughput of a little over $0.5 \mathrm{bits} / \mathrm{channel}$ use between an extreme noise limited case (INR $=-100 \mathrm{~dB}$ ) and a strongly interference limited case (INR $=+30 \mathrm{~dB}$ with a single interferer. (The interference limited case gives a higher throughput, because the MMSE detector can exploit the resulting correlation of the interference plus noise). Note also that for the case of two interferers the variation is greatly reduced, to only a little over $0.1 \mathrm{bits} / \mathrm{channel}$ use. This suggests that the capacity CDF is primarily determined by the SINR (as well as the MIMO channel model), and other parameters of the interference have a negligible effect, which can easily be accommodated by averaging over the distribution of such parameters.

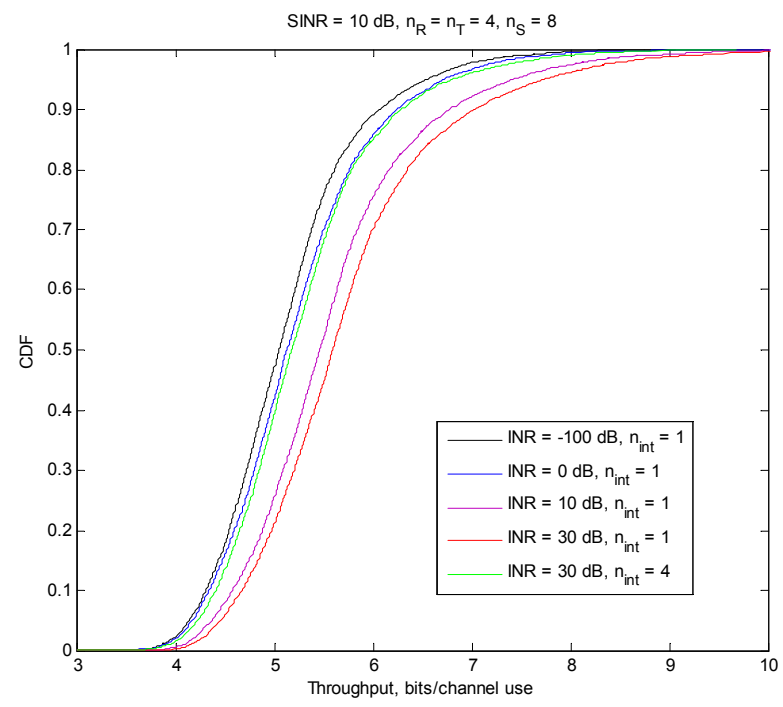

Figure 3 TSB Throughput CDF for SINR $=10 \mathrm{~dB}$, for various SIR and nos. of interferers

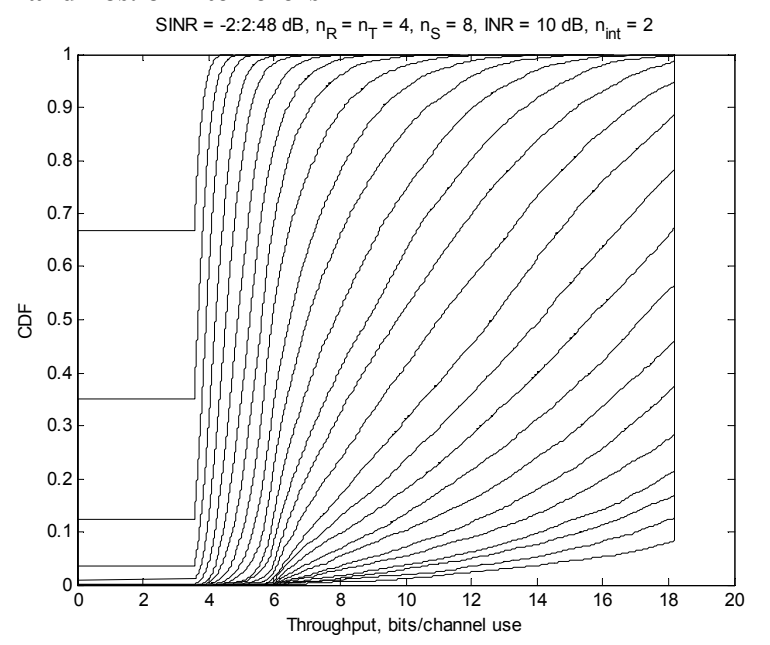

Figure 4 Typical throughput look-up table for use in systemlevel simulation: $n_{S}=8, I N R=10 \mathrm{~dB}, n_{\text {int }}=2$ 
Hence we can derive a look-up table for the link throughput, against SINR and the uniform random variable used to account for random variations. This is illustrated in Figure 4, where the CDF is shown for a range of SINRs from $-2 \mathrm{~dB}$ to $+48 \mathrm{~dB}$ in steps of $2 \mathrm{~dB}$. In a system-level simulator, for each link the SINR would be obtained by calculating signal and interference path losses using an appropriate propagation model (incorporating also shadow fading, etc). Then for each link a uniformly distributed random variable in the range $[0,1]$ would be generated, used to determine the position on the CDF axis, whereupon the link throughput may be obtained by looking up the curve for the appropriate SINR. Of course only a relatively small number of points on each curve need be stored: intermediate values can be obtained by interpolation.

\section{CONCLUSIONS}

We have outlined a methodology to be used in systemlevel simulation to obtain statistically accurate random values for link throughput as a function of SINR for MIMO links, without requiring the full simulation of individual links. This method has been used in system-level simulations in the BuNGee project [10]. It makes use of the truncated Shannon bound to estimate throughput of adaptive modulation and coding schemes. We consider an example based on MMSE MIMO detection in the presence of interference, and show that while the SIR and the number of interferers does affect the throughput distribution, this effect is small compared to the random variations of throughput for given SINR, and hence we conclude that SINR is a sufficient parameter to define the distribution of the throughput. Link throughput can therefore be defined by a two dimensional look-up table indexed by SINR and by a uniformly-distributed random variable on $[0,1]$.

Further work is required to extend and verify the methodology for other MIMO transmission schemes, especially for precoded transmission. In this case in addition to the range of AMC schemes available, there is also a range of precoding matrices available at the transmitter, and this may need to be taken into account in the truncated Shannon bound. The effect of SIR and number of interferers also needs to be checked in such cases.

\section{ACKNOWLEDGEMENTS}

The work described here was funded by the European Commission Framework programme under project BuNGee "Beyond Next Generation Mobile Networks", contract no. 248267. It was also carried out within the framework of COST Action IC1004 "Cooperative Radio Communications for Green Smart Environments”.

\section{REFERENCES}

[1] Gilhousen, K.S.; Jacobs, I.M.; Padovani, R.; Viterbi, A.J.; Weaver, L.A., Jr.; Wheatley, C.E., III; , "On the capacity of a cellular CDMA system," IEEE Transactions on Vehicular
Technology, vol.40, no.2, pp.303-312, May 1991, doi: 10.1109/25.289411

[2] Burr, A.G.; , "Bounds and estimates of the uplink capacity of cellular systems," 1994 IEEE 44th Vehicular Technology Conference, pp.1480-1484 vol.3, 8-10 Jun 1994 doi: 10.1109/VETEC.1994.345341

[3] K. Brueninghaus, D. Astely, et al. "Link Performance Models for System Level Simulations of Broadband Radio Access Systems" IEEE 16th International Symposium on Personal, Indoor and Mobile Radio Communications (PIMRC 2005) vol.4, pp.2306-231, 11-14 Sept. 2005 doi: 10.1109/PIMRC.2005.1651855

[4] J.C.Ikuno, M. Wrulich and M Rupp, "System Level Simulation of LTE Networks," IEEE 71st Vehicular Technology Conference (VTC 2010-Spring), 16-19th May 2010, doi: 10.1109/VETECS.2010.5494007

[5] 3GPP-RAN, LTE - Evolved Universal Terrestrial Radio Access (E-UTRA) Radio Frequency (RF) system scenarios, 3GPP, 36.942 9.0.1, Apr. 2010, release 9.

[6] Caire, G.; Taricco, G.; Biglieri, E.; , "Capacity of bitinterleaved channels," Electronics Letters , vol.32, no.12, pp.1060-1061, 6 Jun 1996, doi: 10.1049/el:19960754

[7] Mariana Goldhammer et al. "Functional BuNGee Specs, Economic model, Energy efficiency and Frequency bands" BuNGee D1.1, see http://www.ict-bungee.eu/page.asp?pID=63

[8] Burr, A.G.; , "Capacity bounds and estimates for the finite scatterers MIMO wireless channel," IEEE Journal on Selected Areas in Communications, vol.21, no.5, pp. 812- 818, June 2003, doi: 10.1109/JSAC.2003.810291

[9] Huaiyu Dai; Molisch, A.F.; Poor, H.V.; , "Downlink capacity of interference-limited MIMO systems with joint detection," IEEE Transactions on Wireless Communications, vol.3, no.2, pp. 442- 453, March 2004, doi: 10.1109/TWC.2003.821168

[10] Tao Jiang et al. "Interim Simulations" BuNGee D4.1.1, see http://www.ict-bungee.eu/page.asp?pID=63 